\title{
ACCESO A DOCUMENTOS E INFORMACIÓN DE LA BANCA CENTRAL EN EL DERECHO COMPARADO: EL CASO DE ESTADOS UNIDOS Y LA UNIÓN EUROPEA
}

\author{
ACCESS TO CENTRAL BANKING DOCUMENTS AND INFORMATION \\ IN COMPARATIVE LAW: CASE STUDY OF THE UNITED STATES AND \\ THE EUROPEAN UNION
}

\author{
Rodrigo GonZÁlez Quintero* \\ Andrés SARMiento Lamus**
}

\begin{abstract}
RESUMEN: El presente trabajo propone un estudio comparado del acceso a documentos e información financiera que repose en la Reserva Federal estadounidense y el Banco Central Europeo. Si bien las regulaciones pertinentes en cada uno de los respectivos sistemas proclama la transparencia, en virtud de las excepciones contenidas en las normas mismas o por medio de las decisiones judiciales, el resultado es una limitación efectiva al derecho a la información financiera ostentado por los ciudadanos.
\end{abstract}

Palabras clave: Banca Central, Derecho a la Información, Derecho Comparado, Estados Unidos, Unión Europea.

ABSTRACT: This paper presents a comparative study regarding access to financial information and documents from the Federal Reserve System and the European Central Bank. Although the relevant provisions in both systems proclaim transparency, yet by virtue of legal exceptions or judicial holdings, the result is an effective restriction over the right to financial information vested on the people.

Keywords: Central banking, Right to Information, Comparative Law, United States, European Union.

\section{INTRODUCCIÓN}

El grado de transparencia de la banca central está directamente relacionado con diversos factores tales como las funciones asignadas a la misma, la precisión operacional de dichas funciones, la claridad de la estrategia en la política monetaria utilizada para lograr sus objetivos, la simplicidad de los instrumentos requeridos para implementar esta estrategia, así como la cantidad, actualidad y calidad de información entregada al público concer-

\footnotetext{
* Profesor-Investigador de la Escuela Mayor de Derecho, Universidad Sergio Arboleda, Bogotá-Colombia. Doctor en Derecho (J.S.D.) por Washington University in St. Louis - Estados Unidos. Dirección Postal: Calle 74 \# 14-14, Bogotá. Dirección electrónica: rgonqui@gmail.com; rodrigo.gonzalez@usa.edu.co

** Profesor-Investigador de la Escuela Mayor de Derecho - Universidad Sergio Arboleda, Bogotá-Colombia. Candidato a Doctor (Ph.D Candidate) por Leiden University - Países Bajos. Dirección Postal: Calle 74 \# 14 14, Bogotá. Dirección electrónica: andres.sarmiento@usa.edu.co
} 
nientes a decisiones de sus órganos de gobierno. ${ }^{1}$ Ahora bien, dicha transparencia dependerá además de la posibilidad de exigir responsabilidad de parte de los ciudadanos a la banca central; y es que, de acuerdo con Apel, "en una democracia, la banca central, como toda institución pública, ha de responder ante la ciudadanía, bien directa o indirectamente"2.

Entendido así como una estructura de responsabilidad, en Estados Unidos el Sistema de Reserva Federal (Federal Reserve System) es supervisado y responde, en principio, ante el Congreso como órgano que en teoría representa los intereses del pueblo estadunidense; en ese sentido, por vía legislativa el Congreso tiene poder sobre la FRS. De otro lado, el Banco Central Europeo responde ante los ciudadanos de la zona Euro; el BCE le reporta al Parlamento Europeo, aunque sus atribuciones estén consagradas en los tratados de la Unión y solo a través de un cambio en ellos pueden afectarse sus funciones. Es por ello que el Parlamento Europeo solo supervisa de manera que puede tan solo "presionar" y no "obligar" al BCE, el cual tampoco es responsable ni ante gobiernos o parlamentos nacionales ${ }^{3}$. Por tanto, en los términos planteados, la banca central europea y estadounidense son responsables solo ante instituciones políticas representativas, en efecto, de alto nivel; y así, de alguna manera dicha responsabilidad estaría diluida ante los ciudadanos.

\section{EL ACCESO A LA INFORMACIÓN DEL SISTEMA DE RESERVA FEDERAL EN ESTADOS UNIDOS}

1. Observaciones generales: la Reserva Federal y la ley de libertad de INFORMACIÓN

Después de ocurrida la crisis financiera del año 2008, la Reserva Federal de Estados Unidos aumentó su rol como actor financiero ${ }^{4}$, determinando aquellas compañías que por tamaño podrían colapsar y, en consecuencia también, las acciones que habrían de implementarse para asegurar la supervivencia de ciertas empresas; en general, tales actuaciones han tenido lugar sin mayor supervisión, en procesos que se debaten en menos de cuarenta y ocho horas. Aunque en el presente comentario se hará referencia a decisiones judiciales que han resuelto el enfrentamiento existente entre el derecho a la información de los ciudadanos y el deber de reserva que asiste a la banca central, cabe adelantar que las actuaciones concretas realizadas por la Reserva Federal en las circunstancias antes descritas, llevaron a que dos organizaciones mediáticas - Bloomberg y Fox- demandaran a la Junta de Gobernadores de la susodicha Reserva (JGFRS), reclamando acceso a los archivos que contenían la información con respecto al rescate financiero, invocando la Ley de Libertad de Información (Freedom of Information Act, FOIA) ${ }^{5}$.

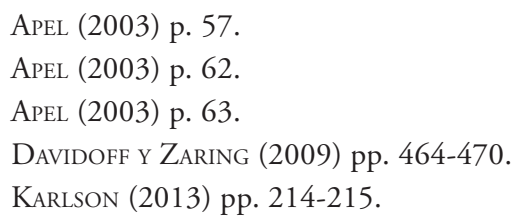


En decisiones judiciales recientes, los tribunales federales se han pronunciado respecto del acceso a la información detentada por la Reserva Federal, invocando el cumplimiento de la FOIA; ${ }^{6}$ pese a que en la sentencia Bloomberg $v$. Board of Governors of the Federal Reserve System $(2010)^{7}$ la Corte Federal del Segundo Distrito ordenó la divulgación de ciertos documentos, y en la sentencia Fox v. Board of Governors of the Federal Reserve System $(2010)^{8}$ la misma corporación ordenó a la JGFRS realizar un barrido adecuado de documentos y solo después del mismo determinar su divulgación o no, otras decisiones del año 2011 -que se expondrán más adelante- expedidas por cortes federales en Washington D.C., han sostenido la reserva de ciertos documentos de la misma entidad.

La interacción entre el sistema de la Reserva Federal y la FOIA, puede enfocarse a partir de que esta última fue promulgada para asegurar la entrega de información que afecte al público; y en ese orden de ideas, los archivos resguardados por los Bancos de la Reserva Federal pueden considerarse como información que afecta al público'. Aunque estos últimos no han sido declarados judicialmente como agencias que han de sujetarse a la FOIA, un examen de sus funciones permite que puedan considerarse como tal. En ese sentido, la referida ley establece el derecho de acceso público a la información relativa a la rama ejecutiva del poder público a nivel federal; en otras palabras, otorga al ciudadano la oportunidad de saber lo que hace el gobierno y asegura una ciudadanía informada, y aunque permite -mediante nueve excepciones- a la agencia cuestionada la negativa a entregar información, atribuye también al ciudadano la revisión judicial de dicha negativa ${ }^{10}$.

En un sistema jurídico de common law, como lo es el estadounidense, el Derecho es aquello que dicen los jueces ${ }^{11}$, es decir jurisprudencial; por tanto, más allá del texto de la norma, hay que atender a los pronunciamientos judiciales. En lo concerniente a la FOIA, los tribunales han realizado una aproximación liberal hacia ella, presumiendo al máximo su espíritu de apertura de la información, profundizando y ampliando dicha intención; sin embargo, la misma consistencia judicial no ha sido posible respecto a la definición del estatus de agencia. Dicho de otro modo, mientras que bajo la FOIA se ha liberalizado el acceso a la información, por el contrario no ha sido posible llegar a un concepto uniforme de aquellos entes públicos sujetos a la norma, por lo que las cortes de justicia han evitado entregar dicha definición absoluta ${ }^{12}$.

Pese a la presunción a favor del acceso a la información, ayudada e impulsada por la judicatura federal, las agencias federales pueden retener información siempre que se

\footnotetext{
Freedom of Information Act, Foia, 5 U.S.C. $\$ 552(1966)$.

BloomberG, L.P. V. BD. of Governors of THE FED. RESERVE SYS., 601 F3d. 143 (2010).

8 FoX NEWS NETWORK, L.L.C., V. BD. OF GOVERNORS OF THE FED. RESERVE SYS, 601 F3d. 158 (2010).

KARLSON (2013) p. 219.

KarLSON (2013) pp. 223-225.

1 MARBURY V. MAdisOn, 5 U.S. (1 Cranch) p. 137.

2 Karlson (2013) pp. 225-227.
} 
demuestre que alguna de las nueve excepciones aplica al caso concreto; la Junta de Gobernadores de la Reserva Federal usualmente invoca las excepciones 4 y 5 -concernientes a secretos transaccionales o información comercial confidencial (5 U.S.C. $\$ 552$ (b) 4) y memorandos internos-externos de agencias (5 U.S.C. $\$ 552$ (b) 5) - con la intención de evitar discusiones abiertas sobre políticas públicas. ${ }^{13}$

\section{LAS SENTENCIAS RELATIVAS AL SISTEMA DE RESERVA FEDERAL Y LA FoiA:}

\section{a. McKinley v. Board of Governors of the Federal Reserve System (Corte Federal de Distrito, Distrito de Columbia, 2010)}

El caso surge a partir de la solicitud de difusión de información hecha por un ciudadano, de acuerdo a la FoIA, a la Junta de Gobernadores del Federal Reserve System (en adelante JGFRS); la demanda buscaba documentos relativos a la autorización de la JGFRS al Banco de la Reserva Federal de Nueva York (en adelante BRFNY), para extender un crédito a JP Morgan que habría de ayudar financieramente a Bear Stearns Companies. La JGFRS en efecto entregó algunos documentos, pero invocando las excepciones 5 y 8 de la Fora retuvo otros, entre los que se encontraba toda la información concerniente a la situación financiera de Bear.

En marzo del año 2008 la JGFRS tuvo conocimiento sobre problemas potenciales de liquidez de Bear Stearns -empresa de holding compuesta por varias entidades financieras- con la certeza que su caída tendría consecuencias impredecibles, pero de seguro severas, para el funcionamiento de los mercados financieros. Sin embargo, dado que Bear Stearns no era institución de depósito, y por tanto no podía acceder a financiación directa de la BRFNY, la JGFRS acordó como mejor opción disponible entregar financiación de emergencia a través de un arreglo con JP Morgan: se autorizó al BRFNY para extender un crédito a aquel, por el que le daría a Bear Stearns un préstamo temporal para asumir sus obligaciones financieras. Así se evitó que la empresa de holding se declarara en quiebra. El demandante requirió de la JGFRS acceso a las minutas de las reuniones y las conclusiones que resultaron en la decisión adoptada; esta respondió entregando varios documentos, pero reteniendo otros en los que se describía información financiera de empresa de holding invocando las excepciones de la FoiA.

La sentencia empieza por describir el Sistema de Reserva Federal, compuesto por la Junta y por doce Bancos Regionales Federales de la Reserva; la Junta supervisa y regula la operación de todo el sistema, promulga y administra regulaciones, y desempeña un papel definitivo en la supervisión y regulación del sistema bancario estadunidense. Si bien cuenta con discreción para examinar libros y cuentas, solicitar informes y reportes sobre activos y pasivos de cualquier banco, no puede extender créditos; los doce Bancos Regionales Federales de la Reserva, por el contrario, si están autorizados para extender créditos $^{14}$.

En su razonamiento, la Corte afirma que la "ForA representa un balance que el Congreso busca entre el derecho del público a saber, y el interés legítimo gubernamental para

13 Karlson (2013) p. 230.

14 MCkinley V. BD. of Governors of the Fed. Reserve Sys., 744 F.Supp. 2 d 128 (2010), p. 132. 
mantener cierta información como confidencial”. Y este interés legítimo se plasma en las nueve excepciones de la FOIA, las cuales "aunque han de ser interpretadas restrictivamente, debe también dárseles la debida aplicación y significado"; por lo tanto, "la justificación de la entidad pública para invocar las excepciones de la FoIA, es suficiente siempre que aparezca como lógica o plausible" 15 .

El punto central de los fundamentos jurídicos está en que la JGFRS invoca la excepción 5, en relación al privilegio que cubre al proceso deliberativo; la excepción extiende el privilegio a documentos que reflejen opiniones consultivas y recomendaciones, así como aquellas deliberaciones que reflejen procesos en que decisiones y políticas gubernamentales son formuladas. Tal privilegio se fundamenta en una conclusión obvia, "el que los funcionarios no se expresarían tranquilamente entre ellos, si cada opinión estuviese sujeta a escrutinio judicial o mediático", y así se intenta promover la calidad de las decisiones gubernamentales. Es más, "la calidad de las decisiones sería erosionada seriamente si las agencias estuviesen forzadas a operar en una vitrina o acuario, puesto que se haría imposible el intercambio libre y franco de ideas en asuntos legales o de políticas"16.

En cuanto a la aplicabilidad del privilegio deliberativo, este opera siempre que el material sea 'predecisional' y 'deliberativo'; es predecisional si se genera antes de la adopción de cierta política y la carga de la prueba se atribuye a la agencia, de manera que debe demostrar la existencia del proceso deliberativo y también la influencia de los documentos en tal proceso. Como regla general, el privilegio deliberativo no cubre a las partes del documento que se refieran a hechos, salvo que estos revelen las deliberaciones protegidas. En el presente caso la Corte se declara persuadida por la Junta, en cuanto ha establecido adecuadamente que entregar contenidos documentales sobre hechos, revelaría sus procesos deliberativos. Está además convencida de que "la entrega de la información retenida revelaría el proceso deliberativo de la JGFRS, [...] exponiéndola de tal manera que desanimaría la discusión tranquila dentro de la misma y resultaría en la erosión de su habilidad para desempeñar sus funciones" ${ }^{17}$. Es una cadena de resultados: la apertura y entrega de los documentos solicitados llevaría a exponer el proceso decisional de la Junta, consecuencia del cual se desanimaría las discusiones y erosionaría la habilidad de esta para realizar su trabajo.

La Junta invoca además la excepción 8 de la FOIA que permite retener información conectada con reportes de condición u operación, preparados por agencias que son responsables por la regulación y supervisión de instituciones financieras. Según la Corte, la excepción 8 cumple dos propósitos: el primero, garantiza la seguridad de instituciones financieras eliminando el riesgo de publicar evaluaciones y reportes que afectarían la confianza de los consumidores en los bancos. El segundo, proteger la relación entre los bancos y las agencias que los supervisan, pues el acceso libre a los reportes para el público y competidores haría que los bancos fueran renuentes a cooperar con las autoridades. Y si bien es cierto que las excepciones de la FoIA deben interpretarse restrictivamente, también lo es que "el

15 MCKINLEY V. BD. OF GOVERNORS OF THE FED. RESERVE SYS., p. 135.

16 MCKINLEY V. BD. OF GoVERNORS OF THE FED. RESERVE SYS., p. 138.

17 MCKINLEY V. BD. OF GOVERNORS OF THE FED. RESERVE SYS., pp. 138-140. 
alcance de la excepción 8 es particularmente amplio [...], [por lo que] si el legislador ha redactado intencional y claramente una definición amplia e inclusiva, no es función del juez -ni siquiera en el contexto de la FoIA- subvertir dicho esfuerzo"18.

Así, para la Corte es evidente que el proceso de supervisión de la Junta es uno de interacción e información compartida con las entidades reguladas, realizado bajo garantías estrictas de confidencialidad; en el litigio en cuestión, la información recibida en tiempo real contenía la afectación de instituciones financieras ante el eventual colapso Bear Stearns, por lo que hacía parte de los requisitos contemplados en la excepción 8. Por tanto, se concluye que la JGFRS retuvo apropiadamente los documentos, de acuerdo a las excepciones de la FOIA, pues "su capacidad para recolectar la información requerida para lograr su misión de regular nuestro sistema bancario nacional, sin duda estaría seriamente comprometida si tal información fuera entregada" 19 .

\section{b. McKinley v. Board of Governors of the Federal Reserve System (Corte Federal de Apelaciones, Circuito del Distrito de Columbia, 2011)}

La decisión anterior fue apelada, y en dicha sede el tribunal empieza por hacer observaciones acerca del sistema de reserva federal, el cual se compone de la JGFRS y doce Bancos de Reserva Federal (en adelante BRF). La primera ejercita poderes de supervisión significativos sobre los segundos, entre los que se destacan "examinar a discreción cuentas, libros y asuntos de cada Banco, así como requerir declaraciones y reportes que sean necesarios”. Los Bancos, por su parte, están autorizados para prestar dinero a bancos; ahora bien, "en circunstancias inusuales y exigentes, la JGFRS puede autorizar a un BRF prestar dinero a otras instituciones, [...] siempre que el BRF obtenga evidiencia de que la institución no tiene forma de asegurar un crédito de otra institución financiera” ${ }^{20}$.

El tribunal retoma los hechos que dieron lugar a la sentencia apelada, reconociendo a Bear Stearns como partícipe importante de muchos mercados financieros, haciendo énfasis en la naturaleza limitada de los instrumentos con los que la JGFRS podía responder a los problemas de liquidez descubiertos. Para la Junta la situación frágil de los mercados financieros era clara, y con la posición prominente de Bear Stearns el contagio resultante a otras instituciones sería inevitable ${ }^{21}$.

Se refiere luego el tribunal a la FoIA, la cual "ordena a las agencias federales difundir archivos, a menos que estos puedan encuadrarse en alguna de las nueve excepciones". Excepciones que han de interpretarse restrictivamente, de manera que "no se opaque la política básica de que publicidad -y no secreto- es el objetivo dominante de la norma"22. Ahora bien, según el tribunal, la excepción 5 de la FoIA fue promulgada precisamente porque la difusión de cierto material predecisional y deliberativo daña efectivamente el proceso decisorio de una agencia; como tal, la excepción "fue creada para proteger el proceso delibera-

\footnotetext{
18 MCKINLEY V. BD. OF GoVernors of THE FED. ReserVE SyS., pp. 142-143

MCKINLEY V. BD. OF GOVERNORS OF THE FED. RESERVE SYS., pp. 143-144.

MCKINLEY V. BD. of Governors of THE FED. RESERVE SYS., 647 F.3d 331 (2011), p. 333.

MCKINLEY V. BD. of GOVERNORS OF THE FED. RESERVE SyS., pp. 333-334.

22 MCKINLEY V. BD. of Governors of the FED. RESERVE Sys., p. 335.
} 
tivo gubernamental, asegurando que los consejeros pudiesen expresar sus opiniones libremente a aquellos funcionarios que toman decisiones, sin miedo a que sean públicas. En el curso de actividades diarias, las agencias con frecuencia dependen de opiniones consultivas y recomendaciones que hacen parte integral de sus procesos deliberativos; procesos que ser públicos, inhibirían la discusión franca de temas de política y además dañarían la calidad de las decisiones" 23 .

En ese orden de ideas, y siendo deferente para con el Congreso, el tribunal entiende que el privilegio del proceso deliberativo "refleja el juicio legislativo sobre el cual la toma de decisiones administrativas estaría seriamente afectada [...] [de no protegerse] documentos que reflejan desacertada o prematuramente las opiniones de una determinada agencia." Y para el tribunal es claro que su labor no es revisar la decisión del Congreso -en cuanto a las excepciones de FolA- caso por caso, pues "sería imposible para los jueces establecer una regla jurídica que afecte alguna y no toda la información acerca del proceso deliberativo, que pueda ser entregada sin violar la excepción 5; los jueces se verían envueltos en un proceso continuo de estimar y adivinar los efectos adversos de ciertas decisiones, las cuales implican gran variedad y combinación de piezas de información. Ello resultaría en que los jueces podrían en ocasiones eliminar retenciones de información legítimas, afectando la implementación satisfactoria y eficiente de la FOIA" ${ }^{24}$.

Es evidente para el tribunal, confirmando la decisión del ad quo, que la publicación del material retenido afectaría negativamente la habilidad de la JGFRS para desempeñar sus funciones. Sin embargo profundiza al respecto, pues de mostrarse determinada información, las instituciones supervisadas dudarían de la capacidad de la JGRS para mantener la confidencialidad; y ello haría que estas fueran menos proclives a dar a la JGRS información necesaria para que esta pueda asegurar un ambiente financiero robusto y supervisado. Es decir, "limitaría la habilidad de la JGFRS para obtener información futura, enfriando la libre circulación de información entre las instituciones supervisadas y la Reserva Federal"25. Por tanto, sostiene la decisión del ad quo, reconociendo como apropiada la retención de información de la JGFRS.

No obstante, el tribunal menciona la sentencia Bloomberg v. JGFRS emitida por la Corte Federal del Segundo Circuito ${ }^{26}$; allí, se declaró que los archivos sobre préstamos realizados por los doce BRF a bancos en el año 2008, que contenían nombres de los bancos receptores, cantidad del préstamos, fechas de entrega y vencimiento, y las garantías, no podían retenerse bajo la excepción 4 de FoIA. En los alegatos, la JGFRS no invocó el privilegio deliberativo adscrito a la excepción 5 , por lo que no se discutieron argumentos relevantes para el presente caso.

Aunque las dos sentencias reseñadas se dirigen a las excepciones contenidas en FoIA, el comentario a las mismas que se realizará más adelante permite evidenciar un test de pro-

23 MCKINLEY V. BD. OF GOVERNORS OF THE FED. RESERVE SYS., p. 339.

24 MCKINLEY V. BD. OF GOVERNORS OF THE FED. RESERVE SYS., pp. 339-340.

25 MCKINLEY V. BD. of Governors of THE FED. RESERVE SyS., p. 340.

26 Bloomberg, L.P. V. BD. of Governors of THE Fed. RESERVE SyS., 601 F3d. 143 (2010). 
porcionalidad asociado a la legitimidad y razonabilidad de tal norma, así como al balance de los intereses en juego.

\section{EL ACCESO A INFORMACIÓN DEL BANCO CENTRAL EUROPEO}

\section{Observaciones Generales: Derecho a la Información en la Unión Europea}

El acceso a la información en el marco normativo europeo, se fundamenta en disposiciones del Tratado de la Unión Europea, del Tratado de la Comunidad Europea, la Carta Europea de Derechos Humanos, y además en regulaciones de instituciones como el Parlamento, la Comisión y el Consejo. Como principio general -y según lo establecido en los artículos 1 del Tue y 255 del TCE- toda persona residente en la Unión tendrá el derecho para acceder a documentos del Parlamento, el Consejo y la Comisión; tal derecho puede ser limitado por el Consejo, en virtud de intereses públicos o privados; y en todo caso, las instituciones habrán de elaborar reglas claras para el acceso a la información.

La Regulación 1049/2001 (Parlamento y Consejo Europeos) avanza sobre el marco general enunciado en los tratados, y aunque dedica ciertos apartes a la regulación de documentos secretos y confidenciales, el principio del que parte es el de libre acceso a los documentos institucionales. No obstante, el derecho a la información -como regla general- encuentra tres grandes excepciones en el artículo 4 de la Regulación, que habilitan a la entidad para abstenerse de entregar documentos así: en primer lugar, si la publicación erosiona el interés público con relación a la seguridad pública, defensa militar, relaciones internacionales, o política económica, monetaria y financiera de la Comunidad o un Estado miembro; en segundo lugar, si se erosionan los intereses comerciales de personas naturales o jurídicas, procedimientos judiciales, investigaciones o auditorías, a menos que exista un interés público superior para la divulgación; en tercer lugar, si la publicación afectará la capacidad decisoria y procesos adjuntos a la misma de la entidad, a menos que exista un interés público superior para su divulgación ${ }^{27}$.

A pesar de su reconocimiento en los múltiples niveles normativos reseñados, existe un debate respecto a si el acceso de documentos de instituciones europeas es o no un derecho fundamental; y dentro de ese debate, si bien la Corte Europea de Justicia ha reconocido consistentemente la importancia del acceso público a dichos documentos, también se ha resistido a calificar tal derecho como fundamental ${ }^{28}$. Cierto es que los fundamentos normativos del acceso a la información van desde tratados hasta regulaciones institucionales, y en principio aplican a las altas instituciones de la Unión. Pero en desarrollo de esos lineamientos, el Banco Central Europeo ha regulado sus actuaciones al respecto (Decisión 2004/258), valiéndose de todas esas normas disponibles al momento de resolver solicitudes que conciernen el derecho en comento.

\section{Sentencia sobre el BCe y el acceso a información}

El caso resuelto en la sentencia Bloomberg v. European Central Bank (Sala Séptima de la Corte General, 2012) surge a partir de la solicitud realizada por la empresa Bloomberg,

7 KRANENBorg y VOERMANS (2005) pp. 31-33.

28 Kranenborg y VOERMANS (2005) p. 31. 
que opera en Londres, al Banco Central Europeo (en adelante BCE) para acceder a documentos relativos a transacciones derivativas en déficits financieros y en el manejo de deuda pública, aplicados al caso de Grecia. La secretaría del Banco negó el acceso; ante una nueva solicitud, el Presidente y la Junta Ejecutiva del BCE confirmaron la citada negativa, alegando de un lado la protección del interés público relativo a la política económica de la Unión Europea y de Grecia, y del otro la protección de consultas y discusiones internas del BCE. El fundamento normativo invocado para tal acción es la Decisión 2004/258, emitida por el propio Banco, en la que se reglamenta la negación de acceso a documentos siempre que se proteja al interés público (confidencialidad de las decisiones y discusiones de entidades europeas; política financiera, monetaria y económica de un Estado miembro o de la Comunidad), una persona privada, o se haga de acuerdo a normas de confidencialidad de la UE (art. 4). Bloomberg demanda entonces el acceso a los documentos.

Para la Sala, "la evaluación de las excepciones al deber de permitir el acceso a documentos, proviene del principio proporcionalidad"; entonces debe considerarse si -dado que las regulaciones invocadas implican una excepción al derecho para acceder a información- el objetivo buscado al negarse el acceso a los documentos puede alcanzarse incluso si se removieran de estos los apartes que podrían dañar el interés público o que contienen opiniones y deliberaciones. Es decir, si efectivamente el objetivo se cumple mediante el actuar del BCE negando el susodicho acceso ${ }^{29}$. La Sala reconoce que "el derecho para acceder a la información concerniente al BCE, deriva de un marco general de apertura de toda decisión originada en órganos comunitarios" (art. 1 TUE), resaltando que "para lograr un buen gobierno y asegurar la participación de la sociedad civil, todo el aparato institucional de la UE debe realizar sus labores tan abiertamente como le sea posible" (art. 15 Tratado de Funcionamiento de la Unión Europea) ${ }^{30}$. Así, según el Tfue (art. 15) toda persona natural o jurídica residente o registrada en un Estado miembro tiene derecho para acceder a documentos de las instituciones, sujeto solo a principios y condiciones del mismo tratado; principios que son la limitación del citado derecho de acuerdo a intereses públicos o privados, determinados vía legislación o regulación, por el Parlamento y el Consejo respectivamente; también se establece que toda institución debe asegurar que sus procedimientos sean transparentes, de manera que permitan acceso a sus documentos.

En concordancia con dichos principios, la decisión 2004/258 establece el derecho para acceder a información (art. 2), sujeto a las limitaciones allí enunciadas (art. 4) que en suma buscan proteger la independencia del BCE y de los bancos centrales nacionales, y la confidencialidad de algunas de sus actuaciones; por tanto, el BCE está habilitado para negar el acceso a documentos, si considera que se afectarán intereses públicos o privados, o si la información se refiere a opiniones internas que incluyen consultas dentro del Banco o con otros bancos centrales nacionales. Pero la Sala es enfática al afirmar que "puesto que las excepciones implican derogación del derecho a la información y acceso a documentos,

29 BLOOMBERG FINANCE L.P. V. EUROPEAN CENTRAL BANK, Sala Séptima - Corte General, ECLI:EU:T:2012:635 (2012), fundamento 31.

30 Bloomberg FinanCE L.P. V. EuRopean Central BANK, fundamento 39. 
estas deben interpretarse y aplicarse estrictamente" ${ }^{31}$. Por tanto, la interpretación de las excepciones realizada por la Sala concluye que si el BCE rechaza el acceso a un documento “deberá -en principio- explicar por qué la publicación de la información podría específica y efectivamente afectar el interés protegido por la norma. [...] Es más, el riesgo de tal afectación ha de ser predecible razonablemente y no meramente hipotético". Y a este respecto, la revisión judicial de la decisión del BCE sobre rechazar el acceso, "debe partir de un reconocimiento discrecional amplio al Banco, en sus funciones para determinar si la entrega de documentos erosiona o no al interés público". Por ello, la revisión judicial "ha de restringirse a si reglas de procedimiento se cumplieron, si se ofrecen las razones para la negativa, se enunciaron los hechos, y si hubo error manifiesto o abuso de poder" 32 .

La Sala afirma también que las excepciones al derecho "se establecen en términos obligatorios, de forma que el BCE tiene el deber de negar acceso a documentos, si las circunstancias enunciadas en ellas se dan". Dado que la norma no contempla la consideración de un 'interés público desbordante', la mera invocación ciudadana de este "ha de rechazarse por ser irrelevante en el análisis contextual de la excepción”33. Entonces aborda la cuestión de la existencia de un error de apreciación del BCE, al negar el acceso; dirá que los oficios mediante los que el Banco se niega a entregar los documentos, mencionan que para ese entonces "los mercados financieros europeos eran frágiles y estaban inmersos en un ambiente vulnerable, cuya estabilidad se afectaba por la situación económica de Grecia”. Además, existía "depreciación de las inversiones griegas, afectando a inversionistas griegos y europeos"; así, el BCE "no cometió error alguno al evaluar que la entrega de cierta información habría de erosionar específica y efectivamente el interés público, en lo concerniente a la política económica de la UE y de Grecia” ${ }^{34}$, por lo que su decisión fue correcta.

La Sala comenta que -en su artículo 10- la Convención Europea de Derechos Humanos reconoce el derecho a la información, sujeto solo a condiciones y formalidades prescritas por Ley y necesarias en una sociedad democrática para la protección de los derechos de otros, así como para la prevención de entregar información recibida en confianza. Además, la Carta Europea de Derechos Humanos -en el artículo 52- establece que cualquier limitación sobre derechos ha de ser establecida por Ley y respetar la esencia de estos, y que en virtud del principio de proporcionalidad, las restricciones deben ser necesarias y conducentes bien a objetivos e intereses reconocidos por la Unión, o bien requeridas para la protección de los derechos de otros. Por tanto, si bien bajo la Carta "el derecho de acceder a documentos de los organismos de la Unión, es como tal un derecho fundamental especial, [...] el demandante no alega violación de tal derecho especial sino apenas afirma una infracción sobre el derecho general de libertad de expresión, [...] sin explicar tampoco cómo el actuar del BCE infringe dicha libertad"35. Así, la mera negativa de acceso a los documentos no implica violación del derecho; por el contrario, la negativa debe ser una que

\footnotetext{
BLOOMBERG FINANCE L.P. V. EUROPEAN CENTRAL BANK, fundamentos 40 y 41.

2 Bloomberg Finance L.P. v. European CENTRAL BANK, fundamentos 42 y 43.

3 Bloomberg FinanCE L.P. V. EuROPEAN CENTRAL BANK, fundamentos 45 y 46.

${ }^{4}$ Bloomberg FinanCE L.P. V. EUROPEAN CENTRAL BANK, fundamentos 52 y 53.

35 Bloomberg FinanCE L.P. V. EuROPEAN CENTRAL BANK, fundamentos 72 y 73.
} 
no se funde en Derecho. Declara entonces la Sala que "la negativa del Banco buscaba lograr un fin legítimo, el de el interés público que concierne políticas económicas de la Unión y de Grecia" ${ }^{36}$, por lo que la pretensión del demandante es desestimada.

La sentencia de la Sala Séptima acude a la proporcionalidad, invocando los enunciados normativos que se contemplan en diversas disposiciones europeas, de acuerdo con el discurrir jurídico que se aborda en los párrafos siguientes.

\section{COMENTARIO A LAS SENTENCIAS}

De manera contundente la decisión judicial europea citada, dispone la resolución del caso a partir del principio de proporcionalidad de acuerdo al fundamento 31. Las sentencias estadounidenses parecen solo ocuparse de las excepciones contenidas en la norma; pero un análisis profundo de las mismas, revela un juicio de razonablidad y de legitimidad de las normas emanadas del legislativo, así como también aplicación del test de balance de intereses, lo que -en suma-implica reconocimiento del test de proporcionalidad.

Entonces las sentencias presentadas confirman la existencia de un fenómeno, la aceptación de la proporcionalidad como principio general del Derecho por cortes constitucionales y tribunales internacionales ${ }^{37}$; de manera más específica, hablar de derechos humanos es hablar de proporcionalidad, ya que como doctrina ha sido recibida y adoptada en las américas, Europa y Africa ${ }^{38}$. Sería correcto reconocer que es una conquista judicial, pues "la práctica jurisprudencial ha aceptado triunfalmente el principio de proporcionalidad, en donde 'balance' emerge como modelo central en el trabajo de los tribunales modernos" 39 .

\section{PRINCIPIO DE PROPORCIONALIDAD EN EL DERECHO EUROPEO}

En Europa, la preeminencia de la jurisprudencia constitucional alemana dentro del derecho comunitario ha sido un factor esencial en la expansión de la proporcionalidad, y en su adopción por la Corte Europea de Justicia (año 1970) y la Corte Europea de Derechos Humanos (año 1976) ${ }^{40}$. Ahora bien, en Alemania no existe disposición constitucional que establezca la proporcionalidad; ella puede deducirse de las propias limitaciones establecidas en los artículos relativos a derechos, y además de la jurisprudencia de la Corte Constitucional en la que se ha sostenido que los derechos están insertos en nociones de proporcionalidad. Afirmación cuyo significado resulta en cada caso, cuando el tribunal identifica un propósito apropiado y la conexión racional entre este y los medios de la norma limitadora, la inexistencia de medios menos intrusivos, y el balance apropiado entre la limitación del derecho y el beneficio obtenido de esta ${ }^{41}$.

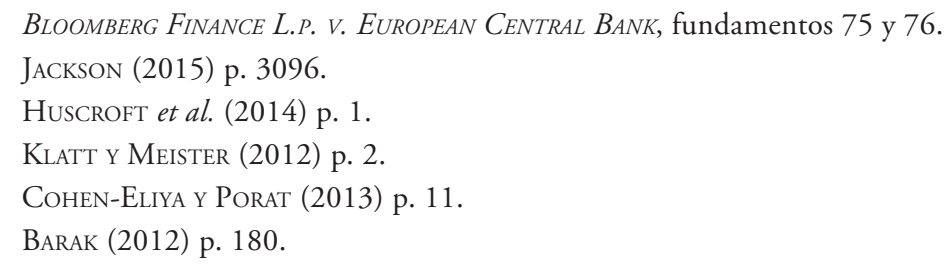


A nivel normativo el principio ha sido consagrado -como la misma Corte General lo evidencia al citar disposiciones tanto de la Convención, como de la Carta- desde el artículo 5 del Tratado de la Comunidad Europea; enuncia entonces la proporcionalidad al ordenar que cualquier acción de la Comunidad no puede ir más allá de lo que sea necesario para lograr los objetivos del tratado, controlando así cualquier instrumento normativo expedido dentro de la Unión y a la vez que opera como el principio judicial ${ }^{42}$.

Proporcionalidad en Europa -bien como principio o bien como test- se erige en una premisa, la inexistencia de derechos absolutos. Así, la gran mayoría de derechos constitucionales gozan solo de protección parcial y no pueden realizarse en su extensión total si su limitación puede ser justificada, pese al consenso sobre que la prohibición de la tortura o la protección de la dignidad humana, sí son derechos absolutos ${ }^{43}$. Proporcionalidad entonces es un instrumento para establecer si la interferencia con un derecho está justificada ${ }^{44}$. Aunque similares, la doctrina ha diferenciado entonces entre su dimensión como principio orientador de la autoridad en su actuación y su dimensión como test aplicable judicialmente. En cuanto principio, comprende cuatro factores que determinan si la restricción sobre un derecho está justificada. El primer factor se refiere a determinar si la actuación de la autoridad que establece el límite busca un objetivo de importancia suficiente para soportar tal restricción; el segundo a si los medios al servicio del objetivo necesario están razonablemente conectados, es decir adecuados, al mismo; el tercero concierne si los medios son aquellos que limitan al mínimo el derecho, así como si se han tenido en cuenta otros medios alternativos que logren el mismo objetivo; y el cuarto se refiere a si los efectos beneficiosos de la limitación sobrepasan los dañinos, es decir, la existencia balance entre el interés público y el derecho ${ }^{45}$.

2. El test JUdicial de proporcionalidad EN EuROpa y EL aCCESO A INFORMACión DEL BCE

El test de proporcionalidad se compone de cuatro elementos o fases, los cuales difieren ligeramente según la jurisdicción, siempre atados a un ejercicio de balance ${ }^{46}$; estos son la legitimidad de los fines del Estado, la adecuación de los medios escogidos para alcanzarlos, la necesidad de los medios escogidos para alcanzar tales fines, y, el balance total de la acción estatal ${ }^{47}$. En sentido estricto el test se orienta a resultados, utilizado cuando el propósito de la norma limitadora es proteger a otro derecho o al interés público; examina el resultado de la norma y el efecto de esta sobre el derecho limitado, comparando el efecto positivo de realizar el propósito de la norma con el efecto negativo de limitar el derecho, es decir, determina si la relación entre el beneficio y el daño es apropiada ${ }^{48}$.

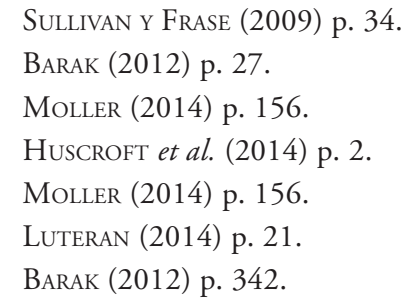


La sentencia de la Sala Séptima realiza - de entrada- una mención específica del principio de proporcionalidad, para luego dirigir el análisis hacia el balance de intereses. Identificará entonces los intereses encontrados como la libertad o derecho de acceder a la información, y el interés público para retenerla. Si bien enmarca ese balance dentro de una esfera normativa de apertura y transparencia en las decisiones comunitarias y además de acceso a las mismas, de igual manera expresa que la normativa permite limitaciones a dicho régimen cuando intereses públicos o privados puedan resultar afectados.

La Sala es enfática en sostener el escrutinio judicial estricto de toda medida que restrinja el derecho a la información; plantea también en abstracto el test de proporcionalidad, de cara a las actuaciones del Banco así: (1) Identifica el interés protegido, pues este ha de explicar por qué la publicación afectaría efectivamente a este; (2) afirma que el riesgo de afectación debe ser razonablemente predecible; (3) la inexistencia de otras medidas, al reconocer amplia discreción al Banco para determinar si el acceso a información afecta o no al interés protegido. Al aplicar el test al caso concreto la Sala opta por una solución elemental, pues afirma que se negó el acceso en virtud de un deber y en ausencia de un interés público desbordante. Y precisamente -dirá- en virtud del principio de proporcionalidad se dan restricciones necesarias, las cuales serán admitidas judicialmente siempre que sean fundadas en Derecho. En suma, acepta la limitación del derecho a la información financiera puesto que se da por un interés legítimo -las políticas económicas de la Unión Europea y de Grecia-, y que no es contraria a Derecho.

\section{El principio de proporcionalidad en la jurisprudencia de la Corte Suprema DE EsTAdos Unidos}

Con respecto a Estados Unidos, pese al rechazo formal, podría alegarse que los niveles de escrutinio estricto adoptados por la Corte Suprema son análogos a estándares derivados de la proporcionalidad ${ }^{49}$; en ese sentido, puesto que el sistema constitucional estadounidense se compromete con un poder público justo -es decir, proporcional- hacia el tratamiento de los ciudadanos, se colige entonces que en confrontaciones para con estos, el Estado está limitado y evita acciones opresivas o $\operatorname{arbitrarias}^{50}$.

En el Derecho estadounidense el principio de proporcionalidad ha sido aplicado expresa e implícitamente por la judicatura, y su raíz común es un juicio en que la intrusión del Estado no ha de ser excesiva en relación con su justificación. El principio puede ser adaptado a cada contexto particular, pues en ciertas situaciones el mayor valor de los derechos en juego justifica una versión estricta de la proporcionalidad, que resulta en el requisito de medios menos restrictivos. En otras, el mayor valor del interés público en juego justifica una versión más ligera de la proporcionalidad, que obliga el Estado a optar por otro medio solo si es sustancialmente menos intrusivo $^{51}$. Se concibe como precepto de justicia -el cual ha sido

\footnotetext{
49 Huscroft et al. (2014) p. 1.

50 JACKSON (2015) pp. 3107-3108.

51 Sullivan y Frase (2009) pp. 169-171.
} 
incorporado en decisiones sobre multas excesivas o castigos crueles e inusuales -que impone límites sobre actuaciones estatales autorizadas, aquellos conectados con justicia y con la idea de prevenir el abuso de poder. También se entiende como aproximación particular estructurada de control judicial, en la que se evalúa si la intrusión sobre el derecho individual puede estar justificada por beneficios derivados de un fin público; esto implica un examen en el que el juez decide si la transgresión está justificada, el cual se conoce como 'el test de balance'52.

Desde hace varias décadas y en temáticas jurisprudenciales muy diferentes -raza, libertad de expresión, o supremacía federal- la Corte Suprema americana ha acudido a categorías que podemos identificar con el principio de proporcionalidad, como lo son el balance de intereses, la razonabilidad y la existencia de medidas menos restrictivas. Así, ha declarado que no existen libertades o derechos absolutos, puesto que la "censura o supresión de la expresión [es] tolerada por la Constitución solo cuando esta presenta un peligro claro e inminente sobre el cual el Estado tiene poder para prevenir y castigar" ${ }^{3}$. Y que "un interés estatal lo suficientemente importante [...] puede justificar limitaciones incidentales sobre las libertades de la Enmienda Primera" 54 .

También ha reconocido la necesidad de balancear intereses enfrentados, como los derechos civiles de un grupo racial específico y la necesidad pública urgente, la libertad religiosa y la unidad nacional, la segregación racial y la igualdad en la educación pública, la libertad de expresión y el interés vital de la Nación en contar con un sistema que funcione con máxima eficiencia para levantar ejércitos que respondan con rapidez y facilidad, o la libertad de expresión y la protección de la integridad de los honores militares ${ }^{55}$. Además, la Corte Suprema ha sostenido a la razonabilidad de normas que regulan el modo, tiempo y lugar de la expresión pública, así como a la verificación de existencia de medidas menos restrictivas para el ejercicio de los derechos ${ }^{56}$.

\section{PROPORCIONALIDAD EN LAS DECISIONES SOBRE ACCESO A INFORMACIÓN DE LA} Reserva FEDERAL

En la sentencia la Corte de Distrito estima que las excepciones de la ley son materialización del balance de intereses, al afirmar que en ella el legislador propende por un balance entre el derecho del público a conocer y el interés del Estado en mantener cierta información como confidencial. Además el tribunal muestra cómo aplicará el test de balance, al anotar que la invocación de las excepciones por parte de las autoridades se tendrá por justificada siempre que sea lógica y plausible. Reconoce además -al referirse a intereses

\footnotetext{
JACKSON (2015) pp. 3098-3099.

W. VA STATE BD. OF EDUC. V. BARNETTE, 319 U.S. 624 (1943), p. 633.

UNITED STATES V. O'BRIEN, 391 U.S. 367 (1968), p. 376.

5 Ver Korematsu V. United StATES, 323 U.S. 214 (1944); W. VA STATE BD. of EDUC. V. BARNETTE; BRoWN V. BD. of EDUC., 347 U.S. 483 (1954); UNITED StATES V. O'BRIEN; UNITED StATES V. ALVAReZ, 132 S. Ct. 2537 (2012).

56 Ver SNyder V. Phelps, 131 S. Ct. 1207 (2011); United States V. Alvarez; United States V. O'Brien.
} 
enfrentados- la prelación de aquellos protegidos especialmente, como lo son los adjuntos al proceso deliberativo pues resulta en decisiones que, a salvo del escrutinio judicial y mediático, revestirán mayor calidad.

Encuentra que la norma es razonable pues las restricciones contempladas son necesarias; aunque las limitaciones a la información han de interpretarse restrictivamente, estas son excepciones consideradas por el legislador y el juez no puede suplantar a este en su discreción. Sumado a la razonabilidad y pertinencia de la medida normativa, el tribunal dispone que la actuación que la actuación de la Junta fue igualmente razonable, pues operó dentro de sus funciones, para proteger información que comprometería el funcionamiento del sistema bancario estadunidense.

La sentencia expedida por la Corte de Circuito -que en gran medida se apoya en lo decidido por la de Distrito- mantiene la razonabilidad de la norma, bajo el entendido que el proceso deliberativo es interés protegido por el legislador. Declina entonces establecer reglas jurídicas caso por caso, pues un segundo examen judicial -por encima del legisladores inviable e impráctico, por lo que la medida es legítima y razonable. Encuentra que los intereses encontrados son el acceso a la información y el desempeño idóneo de las funciones de la Junta; decide limitar el primero, pues la publicación de información delicada podría afectar la robustez del ambiente financiero de los Estados Unidos.

\section{CONCLUSIONES}

En Estados Unidos el marco normativo que determina la relación entre el derecho a la información y la retención de documentos emitidos por la banca central, se circunscribe a la Ley de Libertad de Información (ForA - Freedom of Information Act). Ley cuyo espíritu es el de divulgación, restringido solo por determinadas excepciones, y que se aplica de manera general a todo tipo de agencias federales, entre las que se encuentra la JGFRS. El marco regulatorio de la misma relación en la Unión Europea es mucho más amplio, pues abarca tratados internacionales, directivas y regulaciones; dicha normatividad se dirige a las instituciones principales de la UE, aunque por extensión aplica a otros entes -como el BCEy además a todo tipo de información generada por las autoridades.

Los tribunales federales estadunidenses han entendido que la FoIA plantea un balance de intereses, entre el derecho ciudadano a conocer y el interés estatal de mantener cierta información con el carácter de confidencial; y en cualquier caso, la reserva de información en casos de banca central se ampara en las excepciones de la FOIA, las cuales han de interpretarse restrictivamente. Las excepciones buscan proteger el proceso deliberativo al interior de la agencia, de manera que se promueva la calidad de las decisiones gubernamentales; procesos que incluyen además recomendaciones y consultas recibidas por la JGFRS. Así, se demuestra total deferencia a la norma y a sus excepciones, que a la postre resulta en una doble protección: de un lado, a las funciones de la banca central; del otro, a las entidades cuya información confidencial reposa en manos de la JGFRS. Pese a destacar un ambiente jurídico general de apertura y transparencia institucional en la UE, la justicia comunitaria reconoce que el derecho a la información no es absoluto y por lo tanto admite limitaciones; limitaciones que en el caso del $\mathrm{BCE}$ se orientan hacia la protección de procesos deliberati- 
vos y decisorios. Y ante eventuales ataques, el juez ha de mostrar deferencia hacia los procesos institucionales que retienen información. Entonces, es claro que las sentencias comentadas en el presente escrito -las cuales se han enmarcado en el principio de proporcionalidad y sus elementos adjuntos- sobre la libertad de acceso a información financiera custodiada por la banca central, se han decantado por esta última con la restricción que ella atañe para los derechos de los particulares.

\section{BIBLIOGRAFÍA CITADA}

Apel, Emmanuel (2003): Central Banking Systems Compared: the ECB, the pre-euro Bundesbank, and the Federal Reserve System (New York, Routledge).

BaraK, Aharon (2012): Proportionality: Constitutional Rights and their Limitations (New York, Cambridge University Press).

Davidoff, Steven y Zaring, David (2009): "Regulation by Deal: The Government's Response to the Financial Crisis", Administrative Law Review, Vol. 61, №. 3: pp. 464-541.

Cohen-Eliya, Moshe y Porat, Iddo (2013): Proportionality and Constitutional Culture (New York, Cambridge University Press).

Huscroft, Grant, Millerm, Beadley y Webber, Gregorie (2014): "Introduction”, en Huscroft, Millerm, Grant; Beadley y Webber, Gregorie (edit.), Proportionality and the Rule of Law: Rights, Justification, Reasoning (New York, Cambridge University Press) pp. 1-17.

JACKSON, Vicki (2015): "Constitutional Law in an Age of Proportionality", The Yale Law Journal, Vol. 124: pp. 3094-3196.

KaRLSON, Kara (2013): "Checks and Balances: Using the Freedom of Information Act to Evaluate the Federal Reserve Banks", American University Law Review, Vol. 60, №. 1: pp. 214-270.

Klatt, Matthias y Meister, Moritz (2012): The Constitutional Structure of Proportionality (Oxford, Oxford University Press).

Kranenborg, Herke y Voermans, Wim (2005): Access to Information in the European Union: A Comparative Analysis of the EC and member State legislation (Groningen, Europa Law Publishing).

Luteran, Martin (2014): “The Lost Meaning of Proportionality", en Huscroft, Grant; Millerm, Beadley y Webber, Gregorie (edit.), Proportionality and the Rule of Law: Rights, Justification, Reasoning (New York, Cambridge University Press) pp. 21-42.

Moller, Kai (2014): "Proportionality and Rights Inflation", en Huscroft, Millerm, y Webber, Gregorie (edit.), Proportionality and the Rule of Law: Rights, Justification, Reasoning (New York, Cambridge University Press) pp. 155-172.

Sullivan, Thomas y Frase, Richard (2009): Proportionality Principles in American Law (Oxford, Oxford University Press).

\section{NORMAS CITADAS}

FREEDOM OF INFORMATION ACT, FOIA, 5 U.S.C. $\$ 552$ (4 de julio de 1966).

Regulación del Parlamento Europeo y del Consejo Europeo №. 1049/2001 (30 de mayo de 2001). 
Tratado de la Unión Europea, Tue, art. 1 (7 de febrero de 1992).

Tratado Constitutivo de la Unión Europea, Tce, art. 255 (25 de marzo de 1957).

Tratado de Funcionamiento de la Unión Europea, Tfue, art. 15 (25 de marzo de 1957).

\section{JURISPRUDENCIA CITADA}

Estados Unidos: MARBURY V. MADISON, 5 U.S. (1 Cranch) 137 (1803).

Estados Unidos: W. VA STATE BD. OF EDUC. V. BARNETTE, 319 U.S. 624 (1943).

Estados Unidos: Korematsu V. UNITED StATES, 323 U.S. 214 (1944).

Estados Unidos: BROWN V. BD. OF EDUC., 347 U.S. 483 (1954).

Estados Unidos: United States V. O’Brien, 391 U.S. 367 (1968).

Estados Unidos: BloOmberG, L.P. V. BD. OF GOVERNORS OF THE FED. RESERVE SYS., 601 F3d. 143 (2010).

Estados Unidos: FoX NEWS NETWORK, L.L.C., V. BD. OF GOVERNORS OF THE FED. RESERVE SYS, 601 F3d. 158 (2010).

Estados Unidos: MCKINLEY V. BD. OF GOVERNORS OF THE FED. RESERVE SYS., 744 F.Supp. 2d 128 (2010).

Estados Unidos: MCKINLEY V. BD. OF GOVERNORS OF THE FED. RESERVE SyS., 647 F.3d 331 (2011).

Estados Unidos: SNYDER V. PHELPS, 131 S. Ct. 1207 (2011).

Estados Unidos: United States V. Alvarez, 132 S. Ct. 2537 (2012).

Unión Europea: Bloomberg Finance L.P. V. European Central BANK, Sala Séptima Corte General, ECLI:EU:T:2012:635 (2012). 